\title{
Needle Deflection Studies for Optimal Insertion Modeling
}

\author{
Ka Wei Ng, Jin Quan Goh, Soo Leong Foo, Poh Hua Ting, Teck Kheng Lee, Kesavan Esuvaranathan, \\ Qing Hui $\mathrm{Wu}$, and Edmund Chiong
}

\begin{abstract}
Needle insertion for minimally invasive surgery is a technique explored and studied for percutaneous procedure, diagnosis, localized therapeutic drug delivery, and Biopsy. While the instruments and techniques determine the success of every surgical procedure, minimal attention was given to the medium, the interaction between the tissue and the needle, the development tools and surgical techniques. This paper addresses the interaction by studying the needle deflection during the insertion into porcine back tissue and simulated flesh-like tissue. A customized testing setup is developed to measure and quantify these interactions. The needle deflection magnitude and its insertion characteristics were measured and correlated to define the bio-mechanical properties of back abdomen tissue upon insertion. Needle deflections were measured for gelatine analogues developed to model the consistency of the tissues in the lumbar region of the back. This study was the first step in developing a deflection feedback controlled surgical instrument which enable the needle to reach its intended target in the percutaneous operation. The experimental setup and test procedure provides an understanding on the mechanics of needle insertion, potentially aid the design improvement on surgical instrument.
\end{abstract}

Index Terms-Needle insertion, needle deflection, porcine tissue and gelatine.

\section{INTRODUCTION}

When a bevel-tip needle is inserted into soft tissue, the tip asymmetry will result in the needle bending naturally. The needle may deviate from its intended path due to tissue inhomogeneity and anisotropy, organ deformation, respiration of the patient and etc. Although surgeons and interventional radiologists have long been aware that the tip asymmetry causes needle deflection, manual steering of highly flexible needles remains a challenge due to the limitations of human manual control, lack of access to quantitative tissue models, and complexity of the needle paths [1]. Inaccurate needle placement may result in malignancies not being detected during biopsy, radioactive seeds not being placed in the correct location to destroy cancerous lesions during brachytherapy, and traumatic or even fatal effects during administration of anesthesia. Thus,

Manuscript received May 15, 2013; revised July 21, 2013. This work was supported in part by the Ministry of Education (MOE) Singapore under grant number MOE2010-IF-1-029.

K. W. Ng, J. Q. Goh, S. L. Foo, P. H. Ting, and T. K. Lee are with the Institute of Technical Education, SG Singapore (e-mail: ng_ka_wei@ite.edu.sg, Foo_Soo_Leong@ite.edu.sg, Lee_Teck_Kheng@ite.edu.sg).

E. Kesavan, Q. Wu, and E. Chiong are with National University Hospital Singapore, SG Singapore (e-mail: kesavan_esuvaranathan@nuhs.edu.sg, qing_hui_wu@nuhs.edu.sg, edmund_chiong @ nuhs.edu.sg). for effective medical diagnosis and treatment, the needle must reach its intended target with minimal deviations. Accurate path planning and control of needle steering require models of needle-tissue interaction. Understanding biomechanical interaction between surgical needle and human tissues during needle insertion is crucial to the success of all invasive surgery [2]. Such studies are vital for the design of surgical instruments meant to assist surgeon in specific surgical procedures. DiMaio and Salcudean [3] studies shows that the design of new medical tools without an in depth understanding of the biomechanical properties of simulant or the availability of a realistic simulation model may result in catastrophic consequences, the reliability and applicability of such a tool over a period may also be questionable Hiemenz et al. [4] studies shows that these understandings can prevent the accidental puncturing of vital organs, extensive tearing of skin tissues or inaccurate sampling during biopsy.

Many types of simulants with texture resembling that of human tissues have been made widely available but most remain a mock-up to the human tissues. Example includes the use of ballistic gelatine for firearms [5] and silicone for training simulation. The best replica closest to human tissue is the use of porcine tissue from specific portion of the animals. Such usages are usually costly and morally less acceptable compared to cheaper and widely available simulants.

The most common approach uses a portion of pork as the simulated subject. Alternative, the use of replacement jellifying agents is more convenient, readily available and received better acceptability over cadaver or animal testing. As gelatine is derived from animal collagen, it possessed similar properties and attributes to that of human tissues. Thus, gelatine is an appropriate tissue simulant for needle insertion force testing. In addition, the translucent, yet organic nature of the gelatine allows physical appreciation on the needle deflection during insertion. The formulated gelatine may be used to simulate layers of human flesh for replicating the needle penetration and deflection in the human flesh to reach an internal organ.

Needle deflection affects the accuracy and eventually the duration of surgery [6]. A suitable and standard measurement procedure on the needle deflection profile provides an accurate feasibility study on the usability of a needle guidance medical device.

This paper begins by describing the experimental setup, the procedure and the test samples. Next, the result and discussion of the experiments to characterize the needle deflection during needle insertion into porcine and simulated tissue (gelatine). Thirdly, the results and discussion on the needle deflection against the composition of the gelatine are 
experimented to simulate the invasive surgery. Fourthly, we also tested and compared the mechanical properties of the porcine and the gelatine. Finally, we provide conclusions and recommendations for future works.

\section{SETUP AND EXPERIMENTAL PROCEDURE}

\section{A. Experimental Setup}

A bevel-tip needle, gauge 18, diameter of $1.12 \mathrm{~mm}$ and $15 \mathrm{~cm}$ length is mounted to the digital force measurement gauge. The bevel tip is a cylinder cut through at an angle. It is lowered down to touch the surface of the specimen. Shimadzu's SMX-2000 is an X-ray Fluoroscopic inspection system for observing and inspecting internal structures and mounting conditions in system. Its X-ray system consists of high-resolution flat panel detector produces distortion-free, reliable images. Panoramic imaging process in the system provides a stitching of the multiple images into a more collective view of the insertion. Fig. 1 shows the experimental apparatus.

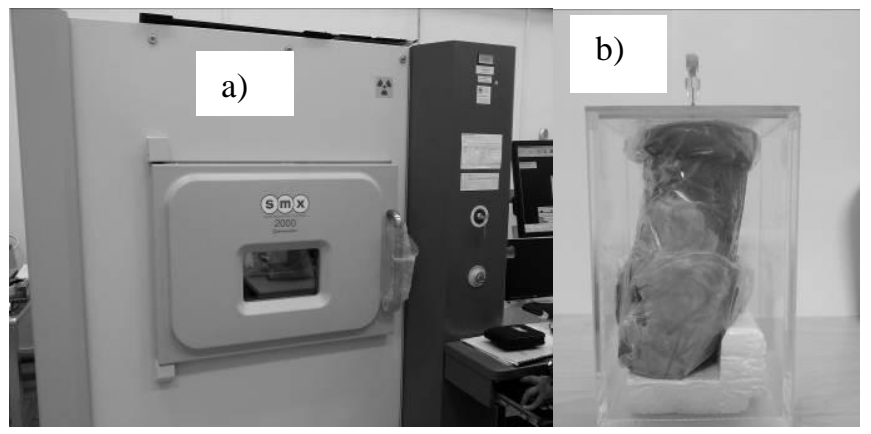

Fig. 1. The needle deflection measurement in tenderloin a) the X-ray equipment SMX 2000 from Shimadzu b) the porcine sample for X-ray imaging with the inserted bevel needle.

\section{B. Experimental Procedure}

In our penetration procedure, the needle is first positioned $1 \mathrm{~mm}$ above the surface of the sample, driven down $80 \mathrm{~mm}$, then stopped and remained still in the prostate for $10 \mathrm{sec}$. We can observe the position of needle by intra operative X-ray fluoroscopy through the cylinder. The displacement of the needle can be visualized and measured by calculating the pixel distance against the reference plane in the X-ray imaging. Other than the displacement, we also use the digital force gauge to measure the insertion force in the experiment. The digital force gauge with a set of needles is attached on a linear stage, and driven vertically at a constant speed of $2.95 \mathrm{~mm} / \mathrm{sec}$. Finally, the needle is withdrawn by $80 \mathrm{~mm}$. Data from the digital force gauge were recorded at a $50 \mathrm{~Hz}$ sampling rate, together with the trigger signal of the linear stage controlled by a computer. The data is analyzed after the experiment. The penetration was performed five times, changing the puncture positions on the surface to prevent the needle from going into the hole created by the previous penetration.

\section{Porcine Tissue Specimen}

Two type of fresh porcine tissue $(100 \times 100 \mathrm{~mm})$ from the back abdomen are used as a closest replacement of human flesh near the kidney area. The fresh porcine tissue $(1 \mathrm{~kg}$ weight each) was prepared and frozen for 2 weeks until the experiment. After 3 days defrosting, it was used for the experiment. The temperature of the porcine specimen at the time of testing was $23.4 \mathrm{C}$.

\section{Preparation of Simulated Tissue Specimen}

Formulated gelatine composition of $57 \mathrm{gm}$ is added to 1.9 liter of water. The gelatine's needle insertion result is compared to a piece of porcine specimen. As gelatine will liquefy when remain at room temperature for more than 30 minutes. Therefore, the duration of experiment is within 10 minutes. The temperature of the gelatine is kept at $4^{\circ} \mathrm{C}$ for the test.

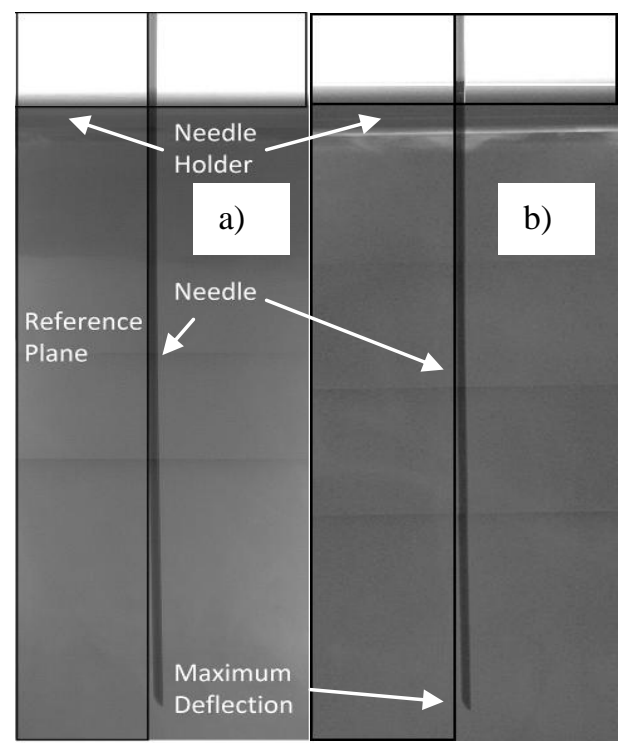

Fig. 2. Visualization of needle deflection under X-ray imaging for the porcine tissue a) tenderloin and $\mathrm{b}$ ) sirloin.

\section{RESULTS AND DISCUSSION}

\section{A. Porcine Specimen Testing}

Fig. 2 shows the X-ray image for the needle insertion into the a) tenderloin and b) sirloin porcine sample. Fig. 2 shows the displacement of the needle tip penetrating into the porcine tissues. Fig. 3 shows the insertion forces acting on the needle for both of the samples. Each force data point was plotted from an average of five samples. The step line in the force plot is the trigger signal of the linear stage controller, and the position of the needle tip. One of the aims of this paper is to model the needle insertion force in the operation conditions.

Interaction between the insertion force and porcine tissue exhibits non-linear properties. The formation of zigzagged force pattern in Fig. 3 implies that the existence of cutting force during the insertion. The cutting force refers to the force that the needle tip slicing through the tissue [7]. We postulate that this force exists as a combination of cutting forces and the tissue stiffness at the tip of the needle. This is due to the needle tip compresses the tissue in front of the cuts. Ideally, the cutting forces will be constant, however from the experimental data in Fig. 3, it shows that the cutting force is inconsistent and it is unrelated to needle depth.

Segregating the sources of the force data into different components, allows accurate modeling of all the individual forces relevant to needle insertion. No design improvement 
of surgical instrument to date was made through explicit monitoring of such individual forces. Plotting force against needle tip position into porcine tissue reveals the variations in friction, cutting forces, and internal stiffness. It also reveals that the collisions of the needle tip with puncture of interior structures. Porcine tissue contains a substantial number of arteries and veins and the internal puncture events are evident in Fig. 3. Summation of the individual force components constitute the overall force data collected. Friction and cutting force are measured after occurrence of primary puncture while stiffness forces measure before needle insertion. Cutting force comprises of plastic deformation of the simulant and the tissue stiffness experienced at the tip of the needle. Internal stiffness of tissue affects the friction force.

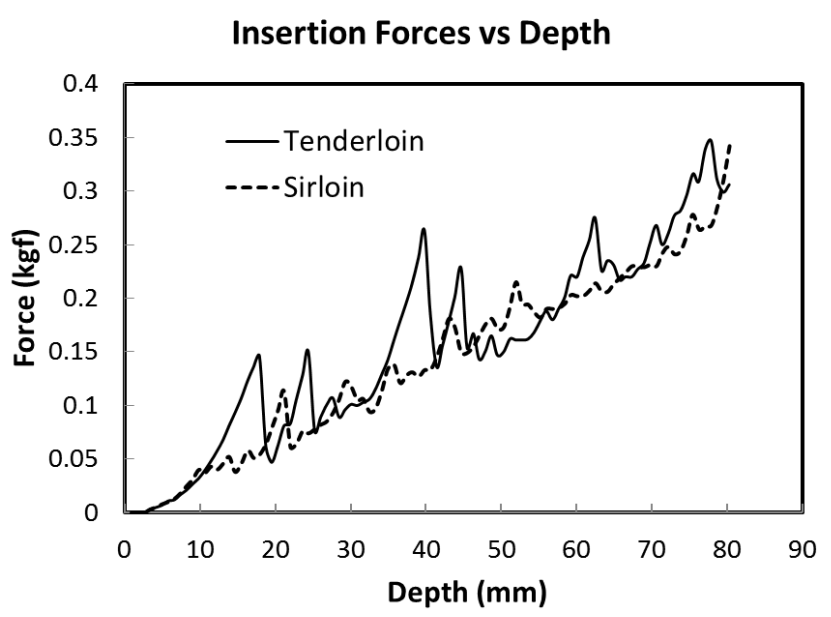

Fig. 3. Insertion force for porcine tissue, tenderloin.

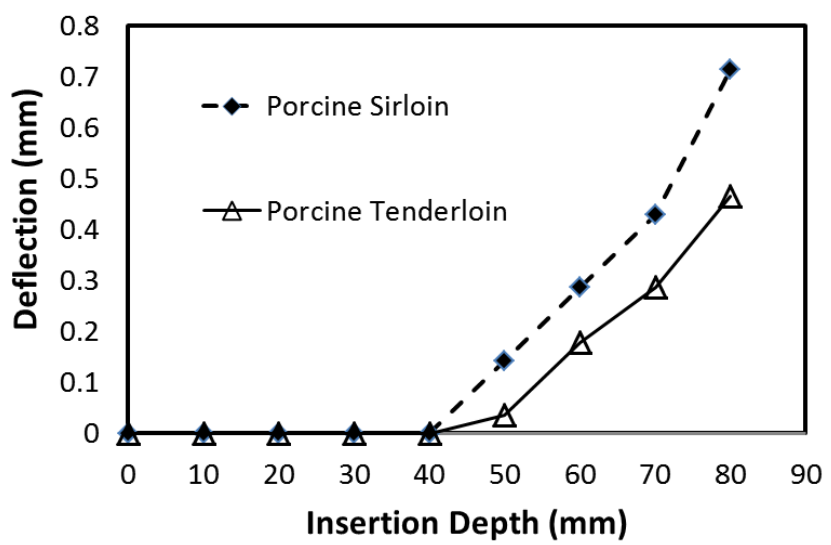

Fig. 4. Needle deflection for porcine samples.

The overall shape of the experimental results for using the porcine tissue (tenderloin) in Fig. 3 is similar to the data for Sirloin, although the significant variations in porcine geometry and internal structure make a perfect match impossible. The differences between these two porcine samples are likely due to variations in small interior structures such as blood vessels. The presence of higher density of blood vessels in tenderloin sample contributed to additional peaks and stiffness forces.

From the needle deflection results in Fig. 4, we can assume that the needle is not completely straight as it travels through the tissue; in reality there are small deflections. Bending of the needle was clearly visible through the X-ray imaging for both of the porcine samples. A less than 1 degree (maximum 0.6 degree) bend was measured from the needle holding point to needle tip. It is clear that tissue deformation is complex, and is still the subject of much research. This is expected as the needle tends to follow the path with less resistance with minimal cutting force. The trials revealed a nonlinear relationship between position and axial force as the needle is inserted. The significant distortion in the insertion path is observed when the depth is $50 \mathrm{~mm}$ or more. This is most likely caused by a large amount of needle bending. A bevel-tip needle, unlike a symmetric-tip needle, will cut tissue at an angle. Since the needle cuts at an angle away from the direction of insertion, the needle may bend in the direction of the bevel. Past experiments have demonstrated the effect of tissue deformations due to rigid needle insertion [6]. Comparison between the tenderloin and sirloin porcine sample indicated that the deflection is different between the two porcine samples. It is believed that the presence of small interior structures such as blood vessels in the tissue provided reinforcement that prevents the deformation of the tissue. The presence of higher density of blood vessels in tenderloin tissue provided additional reinforcement for the tissue to withstand the compression and cutting force instigated by the needle tip and therefore reducing the deflection.

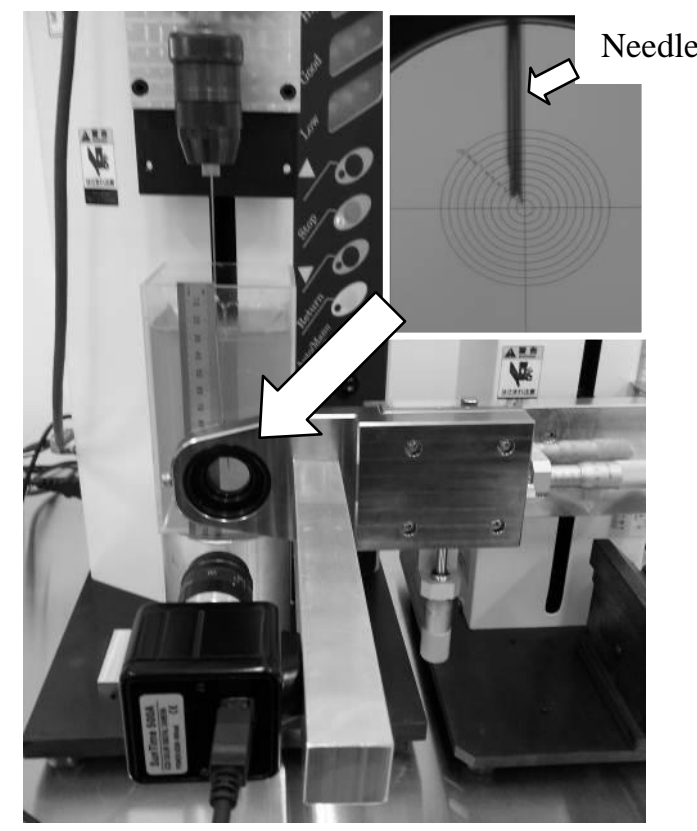

Fig. 5. Vision system setup for needle deflection measurement for gelatin. Inset shows the magnified image of the needle under the camera.

\section{B. Needle Deflection for the Gelatine Samples}

Fig. 5 shows the vision system setup for measuring the needle deflection in the gelatine samples. A camera is used to visualize the needle deflection and the magnitude of the deflection is measured by comparing the axial image position between the needle and the reference plate in the setup. Inset in Fig. 5 shows the magnified image of the needle under the camera. Fig. 6 shows the deflection measurement for the gelatin samples measured using the vision setup. Unlike using porcine sample where the observations are a straight insertion path, a higher increase of deflection was observed. The deflection for gelatine sample is about six-fold higher than the porcine samples (maximum deflection is $4.2 \mathrm{~mm}$ for 
gelatine sample compared to $0.7 \mathrm{~mm}$ for tenderloin sample). An increase in deflections is observed when the gelatine concentration is diluted using water resulting in changes to the viscosity. This increase of the deflection was caused by the increase of the compression force of the gelatine against the needle surface and not by the increase of the friction force between the needle and the gelatine, the result thus tally with the observation that the gelatine surface did not move during the needle insertion. The results also indicate that both insertion and withdrawal have the same pattern of force changes against the composition changes.

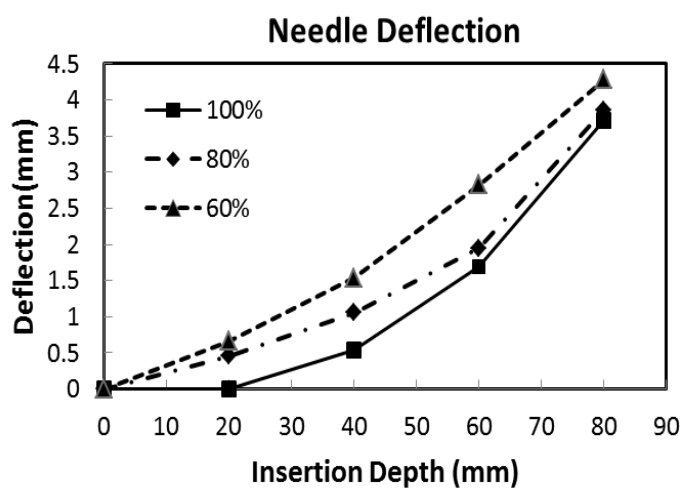

Fig. 6. Needle deflection against the percentage composition of the gelatine.

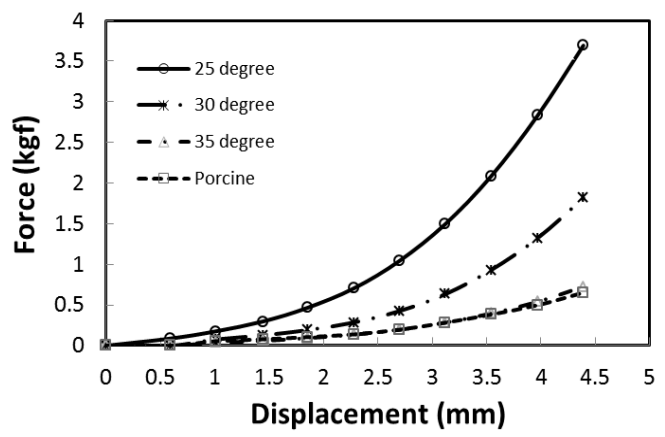

Fig. 7. The comparisons of the compression force of the porcine and gelatine sample under different temperature.

\section{Simulated Fiber Reinforced Gelatine}

In general, tissue modeling is complex because of inhomogeneous, non-linear, anisotropic elastic and viscous behavior [8]. Tissue sample parameters such as Young's modulus (E) and the Poisson ratio (v) are typically determined by testing small homogeneous tissue samples using rheometers and similar materials testing equipment [9]. Measurements of elasticity or the compression forces are usually obtained by loading the tissue sample between two parallel plates. Comparison between the porcine samples and gelatine indicates that the compression force of the gelatine sample is 6-7 times higher than the porcine samples (either sirloin or tenderloin). In order to match the actual biomechanical properties of the porcine sample, we have inserted polymer fiber into the gelatine solution and perform experience with varying the temperature of the gelatine sample ranging from $25^{\circ} \mathrm{C}$ to $35^{\circ} \mathrm{C}$. The comparisons of the compression forces are shown in Fig. 7. It is noted that as the temperature increases to 35 degree, the compression force reduces significantly as compared with the initial condition of 25 degree. It matches the properties of the porcine sample (as shown in the Fig. 7). Using the newly simulated fiber reinforced gelatine sample, the needle deflection measurement is shown in Fig. 8. It demonstrates that the needle deflection for the simulated gelatine resembles the actual porcine experiment.

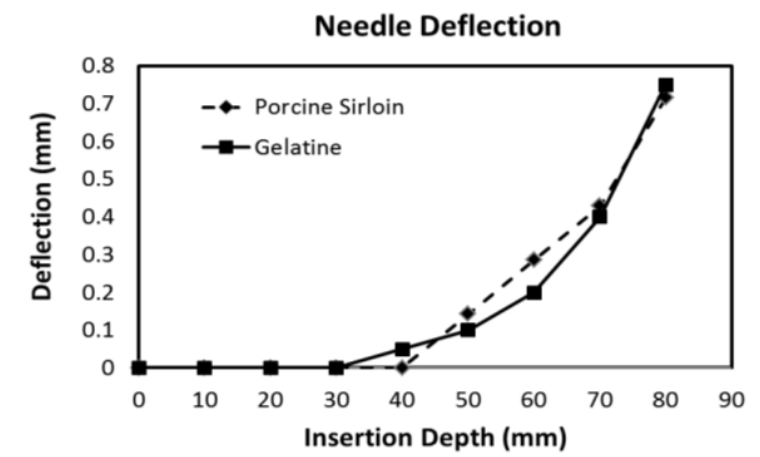

Fig. 8. The comparisons of needle deflection for porcine and gelatine samples.

\section{CONCLUSION}

The needle deflections during the insertion into a porcine tissue were independently measured by a newly-developed test setup, and each deflection was related to the biomechanical behavior of the porcine tissue. The X-ray measurement for the porcine samples' trials revealed a nonlinear relationship between position and axial force as the needle is inserted. The nonlinearity of the deflection during the insertion implies that the compression force between the needle and the medium increase exponentially during the insertion. Additional experiments with the needles insertion for simulated tissue (gelatine) were performed as this jellifying agent is more convenient (transparent in nature which is more suitable for needle defection study), readily available and received better acceptability over cadaver or animal testing. The needle deflection for the simulated gelatine resembles the actual porcine experiment and provides a good alternative medium for design or training of a needle guidance medical device.

\section{REFERENCES}

[1] N. Abolhassani, R. Patel, and M. Moallem, "Needle Insertion into Soft Tissue: A Survey," Med. Eng. Phys., vol. 29, pp. 413-431. 2007.

[2] Y. Kobayashi, J. Okamoto, and M. G. Fujie "Physical properties of the liver for needle insertion control," IEEE/RSJ International Conference on Intelligent Robots and Systems, Sendai, Japan, pp. 2960-2966, 2004.

[3] D. Maio S. P. and S. E. Salcudean, "Needle insertion modeling and simulation," in Proc. IEEE Int. Conf. Robotics Automat., 2002, pp. 2098-2105.

[4] L. Hiemenz, D. Stredney, and P. Schmalbrock, "Development of the force feedback model for an epidural needle insertion simulator," Medicine Meets Virtual Reality 6, pp. 272-277, 1998.

[5] D. S. Cronin and C. Falzon, "Dynamic Characterization and Simulation of Ballistic Gelatine" in Proc. SEM Annual Conference, June 1-4, Albuquerque, New Mexico, USA, 2009.

[6] J. L. Westbrook, D. R. Uncles, B. T. Sitzman, and L. E. S. Carrie, "Comparison of the Force Required for Dural Puncture with Different Spinal Needles and Subsequent Leakage of Cerebrospinal Fluid," Anesth. Analg., vol. 79, pp. 769-72, 1994

[7] C. Simone and A. M. Okamura, "Haptic Modeling of Needle Insertion for Robot Assisted Percutaneous Therapy," presented at the IEEE International Conference on Robotics and Automation, pp. 2085-2091, 2002.

[8] E. Chen and B. Marcus, "Force feedback for surgical simulation," in Proc. the IEEE, vol. 86, pp. 524 - 530, Mar. 1998. 
[9] Y. C. Fung, Biomechanics - Mechanical Properties of Living Tissues, Springer-Verlag, 1993.

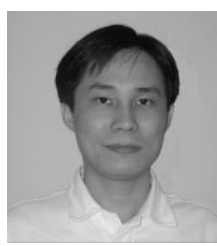

Ka Wei Ng received his M.S. degree from the Nanyang Technological University in Singapore, and his Ph.D. degrees from National University, Singapore, in 2001 and 2010, respectively, all in mechanical engineering. He is currently a Senior Lecturer at Institute Technical Education. His research interests include medical device design, vision system, robotics and automation. Dr. Ng is a member of the American Society of Mechanical Engineers (ASME). He received a 2006 IES (Institution of Engineers, Singapore) Prestigious Engineering Achievement Awards.

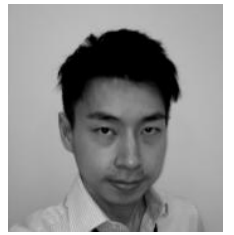

Jin Quan Goh received the B. Eng. degree in mechanical engineering from the Nanyang Technological University in Singapore, in 2005. He was previously from $\mathrm{A}^{*} \mathrm{Star}$, research agency in Singapore, and move on to gain electronic manufacturing experience in Delphi Automotive System. His research interests include medical device design, robotics and automation. $\mathrm{He}$ is currently a Lecturer at Institute Technical Education.

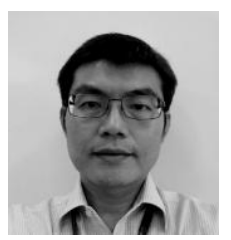

Soo Leong Foo received the B. Eng. degree in mechanical and production engineering from National University of Singapore in 1990 . He had been in the industrial automation sector, working with both local and foreign MNCs such as Matsushita Technology, PSB Singapore, Philips Electronics, NUS for twenty years. His main expertise is in machine system design and development for industries including consumer electronics, semiconductor, aerospace, precision engineering, pharmaceutical and food manufacturing. He is currently the Head for System Integration in the Technology Development Centre for ITE College Central, leading a team of engineers to support the local industry in industrial automation and research projects.

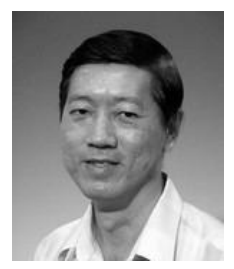

Poh Hua Ting received his Diploma in production technology from German Singapore Institute in 1984. $\mathrm{He}$ has been teaching in the area of Precision Machining/CNC Machining from 1980 to 1987 and Mechanical Engineering from 1988 to 2010. He has worked on a consultancy project with Keppel Ltd in Alignment piping fixture for butt joints from 1999 2000. He was a coach for World Skills Singapore in CNC Turning in 2002, 2004, 2006 and 2008. He is currently a Senior Lecturer in Institute Technical Education

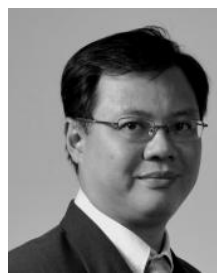

Teck Kheng Lee received the B.S. degree in mechanical engineering and the M.S. degree in materials science and engineering from the National University of Singapore in 1995 and 1999 , respectively and the Ph.D. degree in Mechanical and Aerospace Engineering from the Nanyang Technological University, Singapore, in 2006. He had been with the semiconductor packaging industry for eighteen years, working from the historical package of DIP to the recent flip-chip packages, WLCsp and system-in-package. He joined the Institute of Microelectronics (IME), Singapore, in 1997, researching in the areas of MEMS, CSP, and flip-chip packaging. In 1999, he joined Micron Semiconductor Asia Pte Ltd as a Senior Technical Member, responsible for substrate supplier management and leading some research programs in the field of advanced packaging and material characterization. $\mathrm{He}$ is currently the director of Technology Development Centre for ITE College Central, leading a team of engineers and researchers to support the local industry development in Semiconductor, Automation, ICT and electronics sector. He has authored and co-authored more than 45 papers in journal and international conferences. Currently, he holds 54 U.S patents, pending another 20 U.S patents from issuing. He serves as a committee member for EPTC, SEMI and ICMAT conferences. He is also a member of IEEE, CPMT, Material Research Society (MRS) and IMPAS societies.

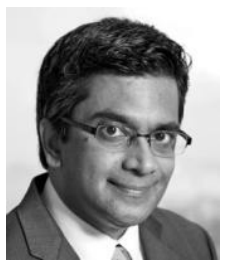

Kesavan Esuvaranathan is the head and senior consultant in the Department of Urology, National University Hospital (NUH). He has more than 25 years of clinical experience in uro-oncology. His research area of interest is in diagnosis and therapy for bladder cancer. He has more than 120 publications and conference papers to date.

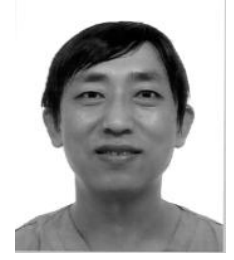

Qinghui Wu received his Ph.D. from National University of Singapore in 2003. Dr. Wu is currently a registrar at Department of Urology, National University Hospital. His research area is in immunotherapy of bladder cancer. Dr. Wu received a Sir Edward Youde Memorial Fund Fellowship in 2002. He received a Young Investigator Award, National Healthcare Group, Singapore in 2005.

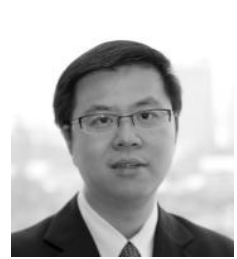

Edmund Chiong received his MBBS from National University of Singapore in 1995, and obtained post-graduate medical qualifications from UK and Singapore. Dr. Chiong is currently a Senior Consultant and Director of Urologic Oncology, Dept of Urology, National University Health System. He is also an Assistant Professor at Dept. of Surgery in the National University of Singapore. His main research areas include investigating immunotherapy, gene therapy and targeted therapy for bladder cancer and new diagnostic modalities for urologic malignancies. He also has research interest in adjunctive therapies for urolithiasis and urinary tract infection. He has received 16 academic awards, including the National Healthcare Group Doctor Award in 2005, American Society of Clinical Oncology Cancer Foundation Merit Award in 2009 and $17^{\text {th }}$ Yahya Cohen Memorial Lectureship Award, Academy of Medicine, Singapore in 2012. He has authored a number of journal publications, book chapters and international conference papers, and has published on novel surface modification methods for medical catheters. He is also a reviewer for more than 15 international scientific journals, several grant funding bodies and the Cochrane Renal (kidney stones) Group. 